\title{
Article \\ Enhanced Proton Acceleration from Laser Interaction with a Tailored Nanowire Target
}

\author{
Yue Chao ${ }^{1,2, *(\mathbb{D})}$, Lihua Cao ${ }^{2,3, * \mathbb{C}}$, Chunyang Zheng ${ }^{2,3}$, Zhanjun Liu ${ }^{2,3}$ and Xiantu He ${ }^{2,3}$ \\ 1 School of Physics, Peking University, Beijing 100871, China \\ 2 Center for Applied Physics and Technology, HEDPS and College of Engineering, Peking University, \\ Beijing 100871, China; zhengcy@iapcm.ac.cn (C.Z.); liuzj@iapcm.ac.cn (Z.L.); xthe@pku.edu.cn (X.H.) \\ 3 Institute of Applied Physics and Computational Mathematics, Beijing 100094, China \\ * Correspondence: litterel@pku.edu.cn (Y.C.); cao_lihua@iapcm.ac.cn (L.C.)
}

check for updates

Citation: Chao, Y.; Cao, L.; Zheng, C.; Liu, Z.; He, X. Enhanced Proton Acceleration from Laser Interaction with a Tailored Nanowire Target. Appl. Sci. 2022, 12, 1153. https:// doi.org/10.3390/app12031153

Academic Editors: Jianxing Li and Ye Tian

Received: 30 December 2021

Accepted: 20 January 2022

Published: 22 January 2022

Publisher's Note: MDPI stays neutral with regard to jurisdictional claims in published maps and institutional affiliations.

Copyright: (C) 2022 by the authors. Licensee MDPI, Basel, Switzerland. This article is an open access article distributed under the terms and conditions of the Creative Commons Attribution (CC BY) license (https:// creativecommons.org/licenses/by/ $4.0 /)$.

\begin{abstract}
Target normal sheath field acceleration via laser interaction with structured solid targets has been widely studied for its potential use in a wide range of applications. Here, a novel nanowire target with a corrugated front surface is proposed to improve the proton acceleration by a target normal sheath field. Two-dimensional particle-in-cell simulations demonstrated that with the existence of the corrugated surface, the cut-off energy of accelerated protons nearly doubles compared to the planar nanowire target. When interacting with the corrugated surface, the incident laser pulse is reflected multiple times, focused and reinforced in each cavity near the front surface, which leads to suppression of the reflectivity and an improvement in the absorption rate. Electrons are heated more efficiently and the sheath field at the target rear side is naturally enhanced. To further investigate the performance of this novel target, a series of simulations with various laser intensities and target sizes were also carried out. This simple target design may provide insights for experiments in the future and should arouse interest because of its wide application.
\end{abstract}

Keywords: target normal sheath acceleration; nano-structured target; laser plasma interaction

\section{Introduction}

With the rapid development of ultra-intense short pulse laser, acceleration of charged particles by laser plasma interaction has become an active area of research due to its prospective application in many areas, such as proton radiography [1,2], tumor therapy [3], isochoric heating [4], tabletop ion accelerators [5], and fast ignition in inertial confinement fusion [6,7]. To obtain high-quality and high-energy ion beams, many acceleration mechanisms have been developed over the past few decades, such as target normal sheath acceleration (TNSA) [8,9], radiation pressure acceleration (RPA) [10-12], and collisionless shock acceleration (CSA) $[13,14]$. Among these mechanisms, TNSA is easy to implement and thus it has been studied widely using both simulations and experiments $[15,16]$. In TNSA, electrons at the front surface of the solid target are heated and accelerated by the incident laser pulse. The hot electrons propagate forward and easily penetrate through the target, establishing an intense charge separation electric field in the vacuum space near the rear of the target. The induced electric field can reach several teravolts per meter, strong enough to ionize and accelerate the hydrogen atoms from water or hydrocarbon contaminants attached on the target surface.

Obviously, the energy and quality of accelerated ions are determined by hot electrons coming from the target front, which are determined by the intensity and absorption of the incident laser. Due to the technological limitations, increasing the laser intensity is very challenging and not practical in experiments, thus improving the energy conversion efficiency from the laser to electrons is of prime importance for TNSA. To achieve this goal, many schemes involving targets with a structured front surface have been proposed, including cone targets [17], double-layer targets [18,19], foam targets [20], nano-structured 
targets [21-23], and micro-tube targets [22,24]. Unlike a planar foil target, the laser pulse is not directly reflected by the sharp density profile, but propagates in the complex geometries of the structured front and interacts for an extended distance with the inner volume of the target. On the other hand, the laser pulse interacting with complex target geometries may benefit the laser absorption via oblique incidence, which provides additional hot electron generation by vacuum heating [25]. The rapid growth of surface micro-structure technologies $[26,27]$ has also facilitated the fabrication of such targets, and many proposed designs have been realized in experiments. Jiang et al. investigated the generation of highquality electron beams from laser interaction with Si microwire arrays [28]. Vallières et al. demonstrated enhanced laser-driven proton acceleration in nanowire targets with different parameters. Dozières et al. studied the optimization of nanowire targets on proton acceleration efficiency [29]. All these works suggest that the performance of nano-structured targets is superior to that of targets with flat surfaces in absorbing laser energies and accelerating hot electrons and energetic ion beams, and ion beams with much higher energies can be generated.

Our study aims to further improve the laser absorption and hot electron generation in a nano-structured target, by tailoring its front surface to modulated geometries [30]. This novel target, known as a corrugated nanowire target (CNT) originates from a planar nanowire target (PNT) and its structure resembles that of the PNT, except that its front surface is tailored so it has a corrugated profile, as shown in Figure 1. Compared to the $\mathrm{PNT}$, the CNT has a larger interaction surface, and more electrons can interact with the laser pulse at the region near the front surface. Furthermore, the laser pulse is obliquely incident in each cavity of the tailored front surface in contrast to its normal incidence in the PNT. This benefits the laser plasma interaction by vacuum heating [25], leading to additional heating of electrons. The oblique incidence also creates multi-reflections of the laser pulse in the tailored structure, meaning that the reflectivity of the laser pulse can be suppressed and more energies are coupled to hot electrons. Here, a series of two-dimensional (2D) particle-in-cell (PIC) simulations were carried out to compare the CNT and the PNT. The simulation results verified that the CNT can significantly enhance the cut-off energies of accelerated protons as well as the energy conversion efficiency. Furthermore, the influence of the laser intensity and the parameters of the corrugated surface on the proton acceleration were examined and are discussed below. The simulation results demonstrated that the proposed target design works well and is superior to the PNT at various parameters, thus providing a robust and simple scheme to obtain protons of much higher energies without requiring a higher laser intensity.
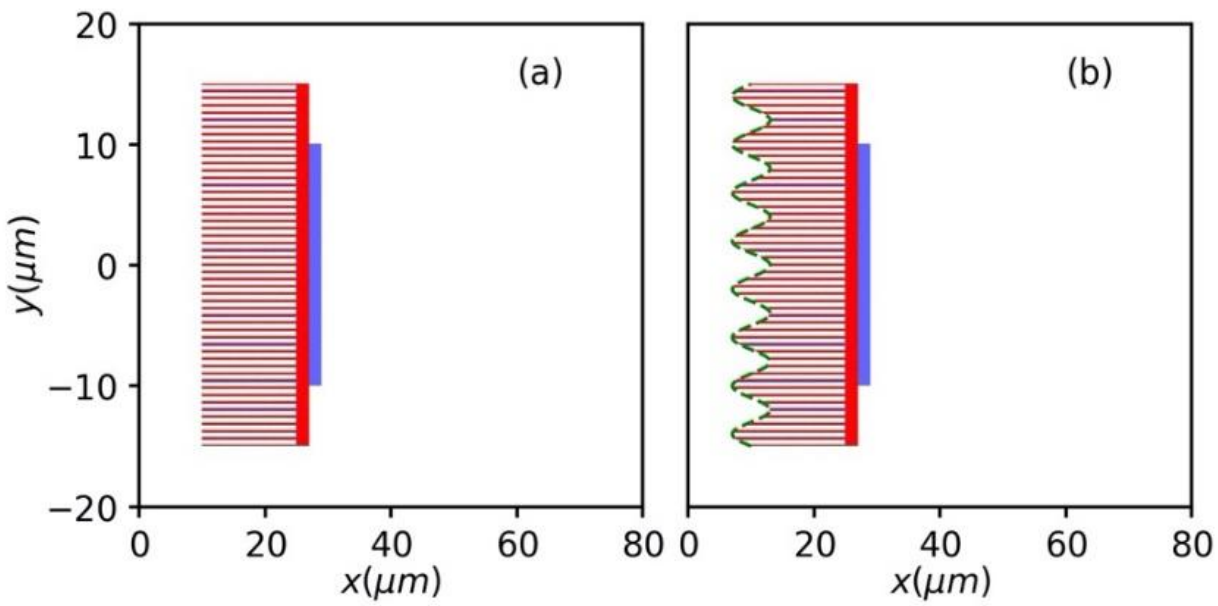

Figure 1. Schematics of the PNT (a) and the CNT (b). Both targets consist of solid nanowires and substrate of $A u^{50+}$ (red part) and a thin hydrogen foil (blue part). The corrugated surface profile is marked by a green-dashed line. 


\section{Simulation Setup}

The two-dimensional simulations were carried out using relativistic PIC code EPOCH [31], which is able to self-consistently simulate the intense laser plasma interaction. The p-polarized laser pulse is incident from the left boundary. It has a flat-top temporal shape with a duration of $15 T_{0}$, including a rising edge of $2 T_{0}$ and a descending edge of $2 T_{0}$. Its peak intensity is $I_{0}=8.6 \times 10^{19} \mathrm{~W} / \mathrm{cm}^{2}$, which corresponds to a normalized vector potential, $a_{0}=8$. The wavelength is $\lambda_{0}=1 \mu \mathrm{m}$, and the period is $T_{0}=3.3 \mathrm{fs}$. The spatial profile of the laser pulse is Gaussian in the $y$-direction, expressed as $I=I_{0} \exp \left(-\frac{y^{2}}{r^{2}}\right)$, with $r=8 \mu \mathrm{m}$. The size of the simulation box is $80 \mu \mathrm{m} \times 40 \mu \mathrm{m}(0 \mu \mathrm{m}<x<80 \mu \mathrm{m},-20 \mu \mathrm{m}<y<20 \mu \mathrm{m})$ with $4000 \times 4000$ grids in the $x, y$ plane. Each cell is filled with 100 electrons, $10 A u^{50+}$ ions and/or 100 protons. The temporal resolution is set as $\tau=0.01 T_{0}$, which is sufficient to accurately calculate the laser plasma interaction. The PNT has a comb-like structure, consisting of three parts, as seen in Figure 1a. The first part is nanowires made of $A u$ with diameters $d_{1}=0.3 \mu \mathrm{m}$, vacuum spacing $d_{2}=0.3 \mu \mathrm{m}$ and length $L=15 \mu \mathrm{m}$. To save computation resources, they are assumed to be pre-ionized $A u^{50+}$ with ion density of $n_{i}=n_{c}$ and a corresponding electron density of $n_{e}=50 n_{c}$. Here $n_{c}=m_{e} \omega_{0}^{2} \epsilon_{0} / e^{2}$ is the critical density, and $\omega_{0}, \epsilon_{0}, e$ are the laser frequency, vacuum permittivity and elementary charge, respectively. According to Hollinger et al. [32], the pre-ionized $\mathrm{Au}^{50+}$ can be obtained in such an intense laser condition, and are adopted in many previous simulation works [33,34]. The second part is a substrate of the same materials with a thickness of $D=2 \mu \mathrm{m}$, to which the nanowires adhere. The nanowires and the substrate are placed in the region $-15 \mu \mathrm{m}<y<15 \mu \mathrm{m}$ and $10 \mu \mathrm{m}<x<27 \mu \mathrm{m}$. The third part is in the rear side of the substrate, where a $2 \mu \mathrm{m}$ thick and $20 \mu \mathrm{m}$ wide hydrogen plasma layer, with $n_{H}=n_{e}=10 n_{c}$, is placed in the region $-10 \mu \mathrm{m}<y<10 \mu \mathrm{m}$ and $27 \mu \mathrm{m}<x<29 \mu \mathrm{m}$. The CNT has the same shape and parameters as the PNT, except that its front surface is corrugated (labeled by a green-dashed line in Figure 1b). The profile of the corrugated surface is modeled by a sinusoidal curve, expressed as $x=10 \lambda_{0}+A \cos (2 \pi K y)$. This configuration ensures that the total number of electrons in the simulation box is basically the same in both targets. A series of simulations were carried out in this work. Firstly, the shape of the corrugated profile was chosen as $A=3 \lambda_{0}$ and $K \lambda_{0}=0.25$. The heating of the electrons and proton acceleration via TNSA were investigated in detail. Furthermore, the parametric dependencies were also studied by changing the parameters of the corrugated profile.

\section{Results and Discussions}

\subsection{Laser Absorption and Hot Electron Generation}

In typical TNSA, the laser pulse irradiates a solid target, usually a foil target. Electrons are heated via vacuum heating and the $\boldsymbol{J} \times \boldsymbol{B}$ mechanism. Hot electrons propagate to the target rear side, establishing an intense sheath field for proton acceleration. Because the laser pulse can only reach several skin depths into the target, a large proportion of energy is reflected. However, for the nanowire target, the laser pulse can propagate in the vacuum gaps between the solid wires, leading to a significant suppression of the reflectivity. Typically, the reflectivity in the nanowire target can be reduced to under $20 \%$ [35]. Furthermore, the laser pulse propagates in the vacuum gaps and interacts with the solid wires, pulling out the electrons and accelerating them via Lorentz force over a long distance [36]. This mechanism makes it possible to generate much more energetic electrons. In the CNT, the tailored front surface is expected to further reduce the reflectivity of the incident laser.

Figure 2 shows the simulation results at $t=22 T_{0}$. To compare the reflectivity, the normalized Poynting flux in the longitudinal direction $\left(S_{x}\right)$ is shown in Figure 2a,b. It can be clearly seen that the reflected component is much weaker in the CNT compared to the PNT. The reflectivity can be estimated by integrating the Poynting flux propagating in the negative direction (blue components in Figure 2a,b, denoted as $S_{-}$) at the left boundary, expressed as $R=\frac{\iiint S_{-}(x=0, y, z) d y d z d t}{W_{0}}$, where $W_{0}$ is the total energy of the incident laser 
pulse. The value of $R$ determines the energy that flows into the target, and the latter corresponds to electron heating and determines the temperature of the electrons. The reflectivity in the PNT is $15 \%$, while in the CNT it is only $5 \%$. This result indicates that in the CNT, more energy is transferred to the electrons in the nanowires, and thus the temperature of the electrons is expected to increase. Furthermore, multiple reflections by the corrugated surface leads to the focusing and reinforcement of the laser pulse in each cavity of the front surface. To better illustrate this, Figure 2d,e show the zoom-in view of $S_{x}$ at the region near the front surface in Figure $2 \mathrm{a}, \mathrm{b}$, and the colormap is also adjusted. It is clearly seen that the intensity of $S_{x}$ is higher in the CNT, and the peak value of the laser electric field is estimated to increase by $50 \%$ comparatively.
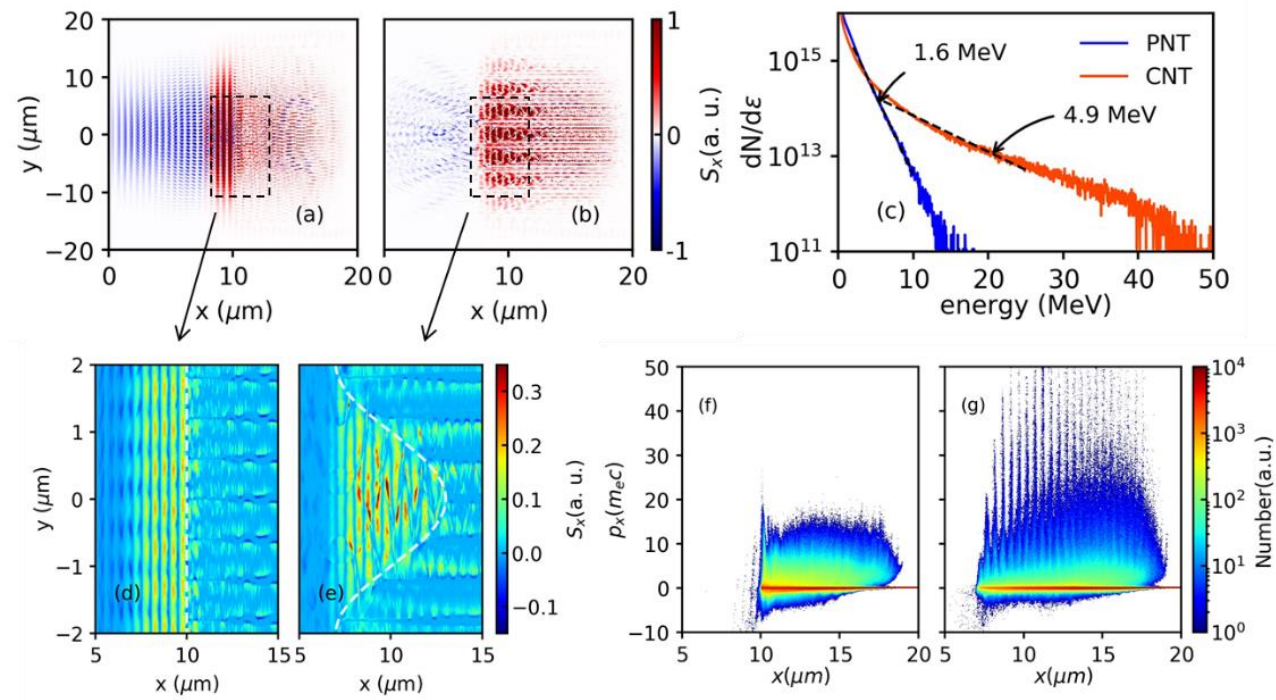

Figure 2. Simulation results at $t=22 T_{0}$. (a,b) Normalized Poynting flux $\left(S_{x}\right)$ for the CNT (a) and the PNT (b), where forward and reflected components are labelled as red and blue, respectively. At this time both incident and reflected components of $S_{x}$ can be observed. (c) Energy spectra of hot electrons in the nanowires. (d,e) Zoom-in view of Poynting flux near the target front surface. $(\mathbf{f}, \mathbf{g})$ Phase space $\left(p_{x}-x\right)$ of electrons in the nanowires.

As discussed above, due to the multi-reflections and focusing of the incident laser pulse by the corrugated surface, the electrons in the CNT are heated more effectively and are able to gain higher energy than those in the PNT. This can be observed from the energy spectra of electrons exhibited in Figure 2c. The spectra are obtained by counting all electrons moving in the $+x$ direction. In the CNT, hot electrons with energies as high as over $40 \mathrm{MeV}$ are generated, compared to a maximum energy of only $15 \mathrm{MeV}$ in the PNT. The energy spectra show significantly different spectra because a portion of low-energy energetic electrons is shifted to higher energies. The temperature of the hot electrons is given by fitting the spectra with a Maxwellian distribution, as labeled by dashed lines in the figure. It increased by as much as 3 times from $1.6 \mathrm{MeV}$ to $4.9 \mathrm{MeV}$. The phase space $\left(p_{x}-x\right)$ of the electrons is also shown in Figure 2g, $\mathrm{f}$, which is consistent with the energy spectra. It is worth noting that the phase space features a periodical structure, and the period of electron bunches is approximately $\frac{\lambda_{0}}{2}$. Similar results have also been demonstrated in previous work $[36,37]$ on nanowire targets. Since TNSA is directly affected by the temperature of hot electrons, an increased temperature will enhance the sheath field established at the target rear surface and the subsequent proton acceleration. This will be discussed in detail in the following section.

\subsection{Sheath Filed and Proton Acceleration}

As hot electrons originating from the nanowires propagate through the target rear side, a strong longitudinal sheath field is established. This is associated with the energy of hot 
electrons $\left(\epsilon_{h} \sim T_{h}\right)$, and the Debye length of hot electrons $\left(\lambda_{D}=\sqrt{\frac{\epsilon_{0} T_{h}}{n_{h} e^{2}}}\right)$. For a solid slab target, the sheath field can be estimated [38] as $E_{\text {sheath }} \sim \epsilon_{h} / \lambda_{D} \propto \sqrt{n_{h} T_{h}}$, where $n_{h}, T_{h}$ are the density and temperature of the hot electrons, respectively. Based on the discussion in the previous section, the energy and number of hot electrons are both increased in the CNT, it is thus expected to generate a stronger sheath field. Figure 3 exhibits the normalized longitudinal electric field at $t=35 T_{0}$. The sheath field in the CNT becomes stronger compared to that in the PNT. Furthermore, it occupies a larger area. Figure $3 \mathrm{c}$ shows the electric field averaged near the axis $y=0$. The enhancement of the sheath field in the CNT is clearly observed, and its peak value is increased from 3 to above 5. As shown in Figure $3 \mathrm{~d}$, the electric potential created by the sheath field is obtained by integrating the electric field presented in Figure 3c, where the right boundary is set as the zero-potential point. The maximum potential in the CNT nearly doubles, meaning that the energy gain in a single proton as it propagates from the target rear side to the right boundary is estimated to double in the CNT compared to that in the PNT.
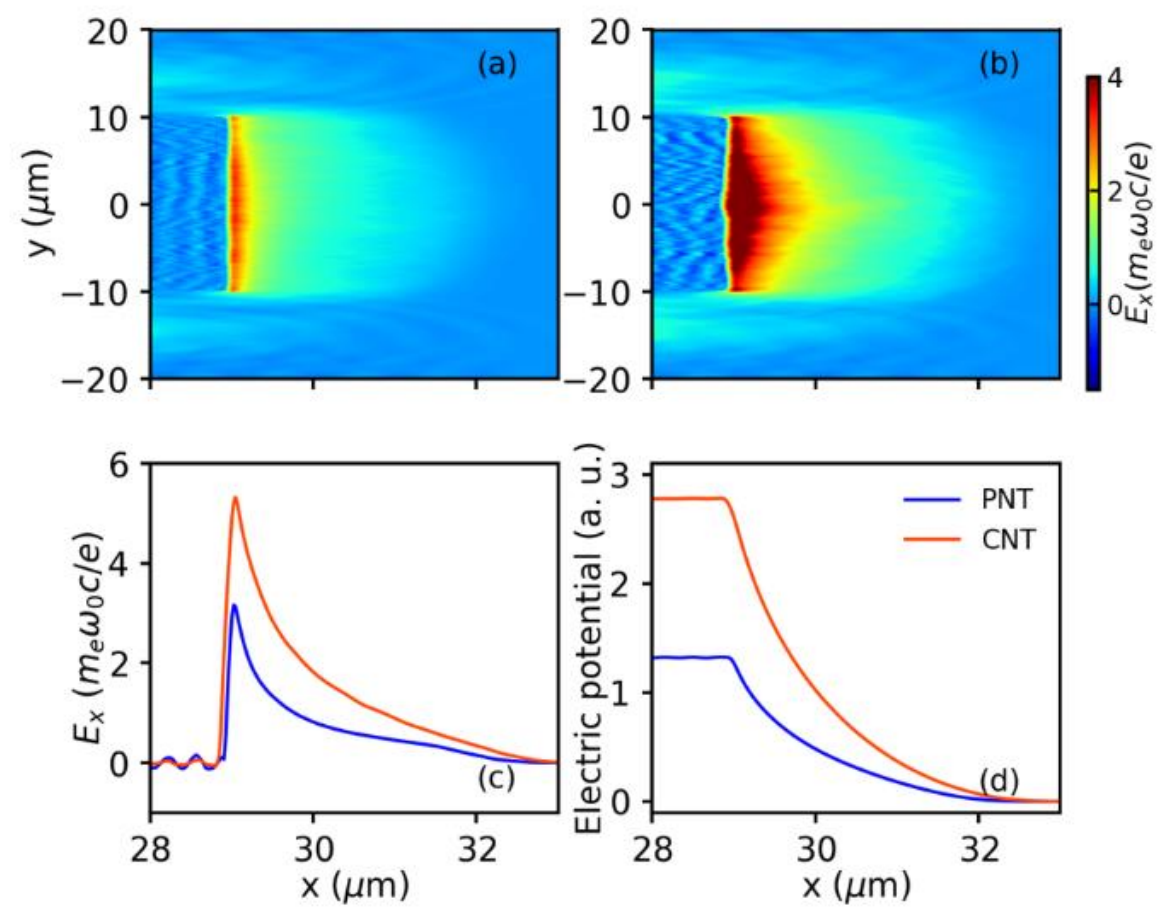

Figure 3. Longitudinal electric field $\left(E_{x}\right)$ at $t=35 T_{0}$ near the target rear surface for the PNT (a) and the CNT (b). (c) Averaged $E_{x}$ near the region $y=0$ for both targets. The electric field is normalized by $m_{e} \omega_{0} c / e$. (d) Corresponding electric potential created by the sheath field near the region $y=0$ for both targets.

Figure $4 \mathrm{a}-\mathrm{f}$ shows the energy density (normalized by $n_{c} m_{p} c^{2}$ ) of protons at different times. One can see that the energy densities of accelerated protons are much higher in the CNT. Besides, the accelerated protons propagate faster in the CNT. These results agree well with the above discussion on the sheath field. The energy spectrum of protons at the end of the simulation is exhibited in Figure 4g. The spectra in both cases feature a typical TNSA shape. In the CNT, the cut-off energy of the protons is about $50 \mathrm{MeV}$, which is much higher than the $22 \mathrm{MeV}$ for the PNT. The proportion of high-energy protons $(>5 \mathrm{MeV})$ is also greatly increased, meaning that more energetic protons are generated in the CNT. To estimate the conversion efficiency from laser to protons, protons with energy greater than $1 \mathrm{MeV}$ were counted, and the laser-to-proton energy conversion efficiency in the CNT was $12.6 \%$, while this value was only $6.7 \%$ in the PNT. 

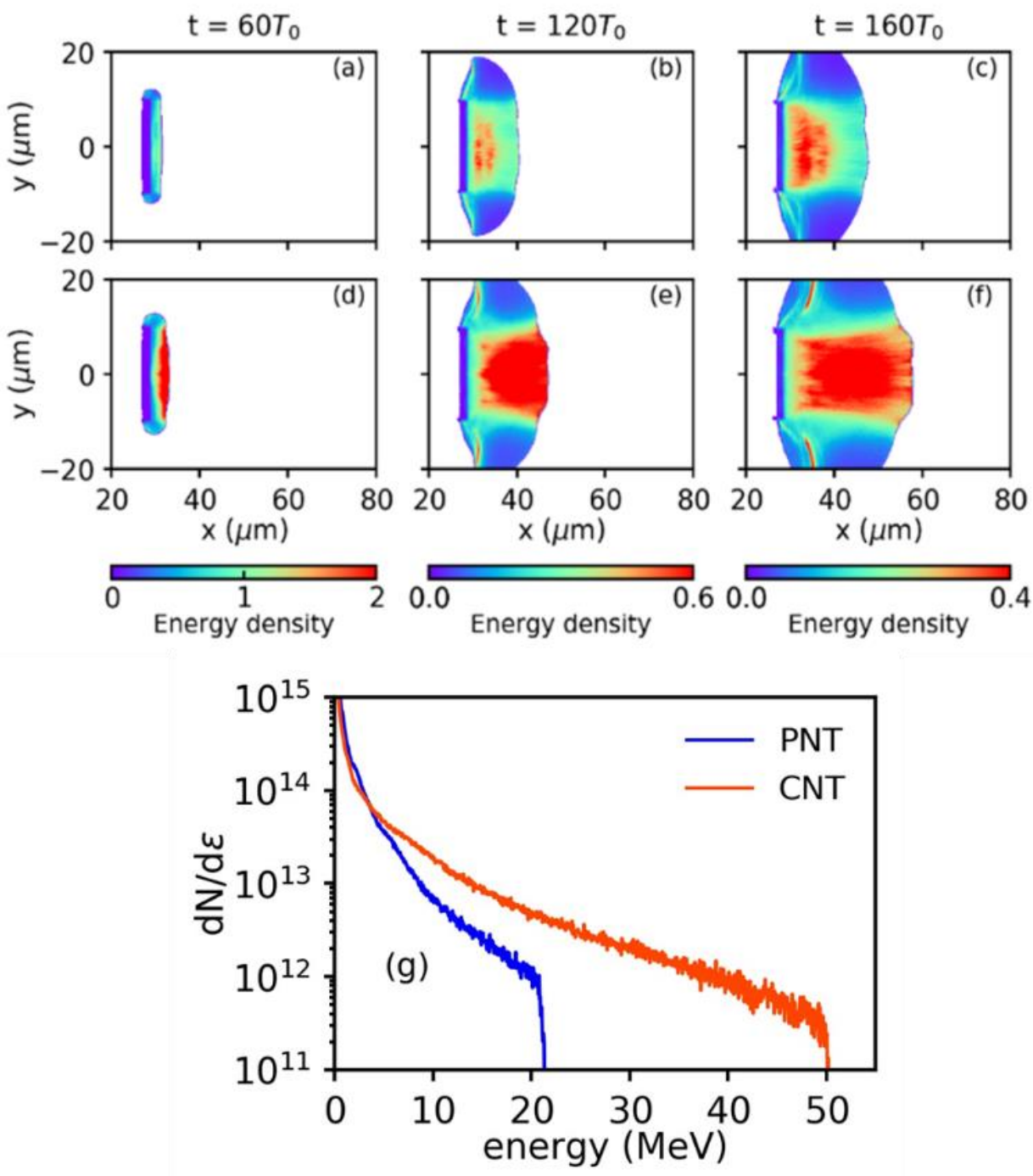

Figure 4. (a-f) Temporal evolution of energy density (normalized by $n_{c} m_{p} c^{2}$ ) at different times for the PNT $(\mathbf{a}-\mathbf{c})$ and the CNT $(\mathbf{d}-\mathbf{g})$ energy spectra of protons at the end of simulation.

\subsection{Robustness of the CNT}

To study the robustness of the CNT, a series of PIC simulations with different laser and target parameters were implemented. Firstly, the amplitude of the corrugated surface (expressed as $x=10 \lambda_{0}+A \cos (2 \pi K y)$ ) was varied from $A=0$ to $A=5 \lambda_{0}$, and $K$ was fixed as $K \lambda_{0}=0.25$, where $A=0$ denotes the PNT. Figure $5 \mathrm{a}$,b show the simulation results. The cut-off energy of the accelerated protons increases with $A$, and the conversion efficiency also increases, meaning that more high-energy protons can be generated with larger $A$. It is worth noting that both the conversion efficiency and cut-off energy tend to converge to a constant value with increasing $A$, as shown in Figure $5 \mathrm{~b}$. This is because the laser reflectivity cannot be suppressed infinitely, and the absorption of the laser pulse also has an upper limit. When $A$ is large enough, the incident laser pulse can be almost totally absorbed, leading to the maximum conversion efficiency and cut-off energy. Figure $5 c, d$ show the cut-off energy of the accelerated protons and the conversion efficiency versus intensity of the incident laser. The cut-off energies of both targets increase nearly linearly with laser intensity, but changes in the conversion efficiency are relatively weak. It is clear that both the cut-off energy and conversion efficiency can be significantly enhanced in the CNT for different laser intensities. 

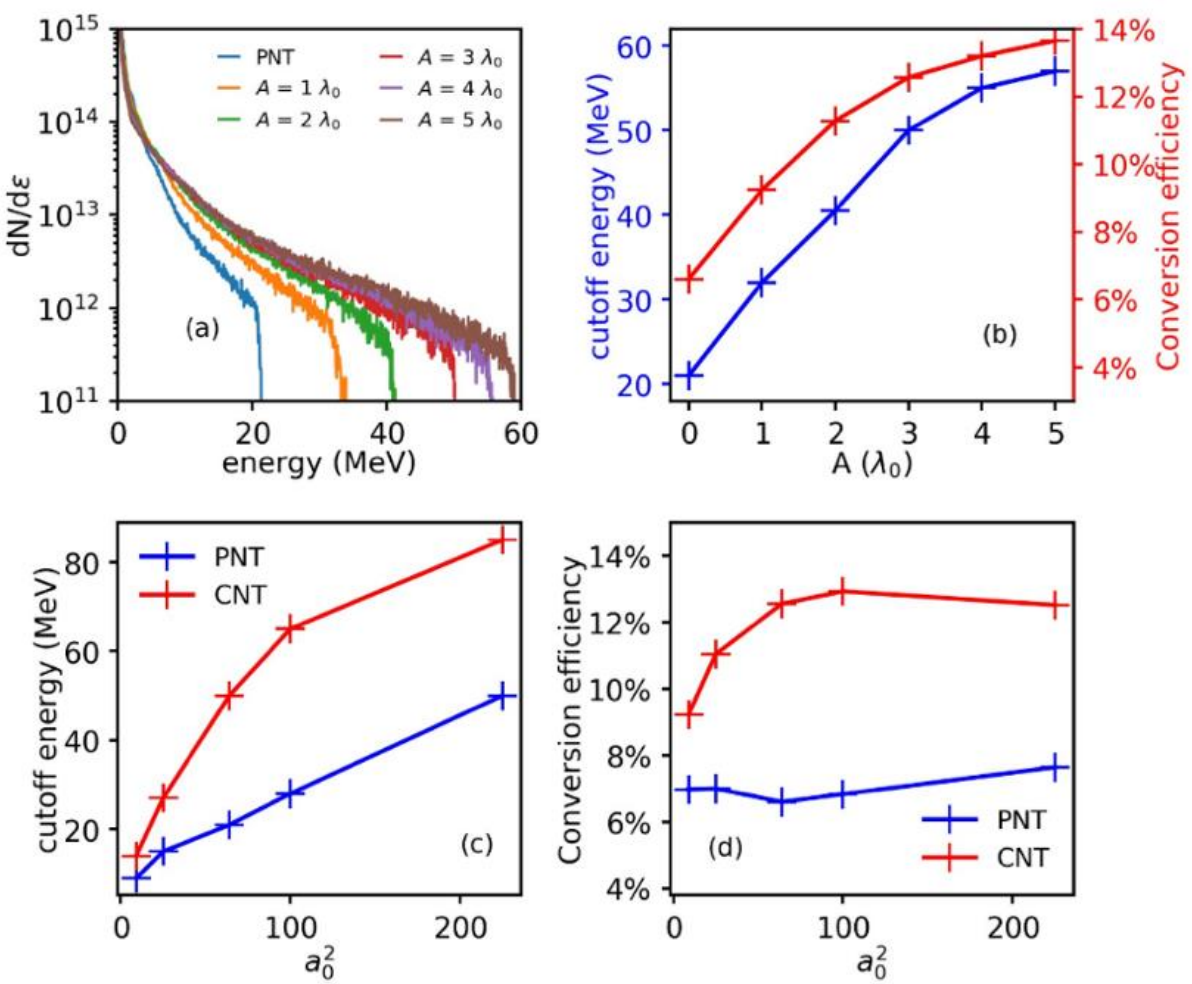

Figure 5. (a) Energy spectra of protons with different $A$. (b) Cut-off energies of protons and conversion efficiency versus $A$. (c,d) Cut-off energies and conversion efficiency versus laser intensity $\left(a_{0}^{2}\right)$ for the $\mathrm{PNT}$ and the CNT, respectively.

In experimental conditions, the laser pulse usually has a long pre-pulse of low intensity. This pre-pulse can pre-heat the target and generate low density plasmas that fill the gaps between the nanowires, which may impair the laser absorption and hot electron generation. Dozières et al. [29] have studied the effect of pre-pulses on laser absorption and TNSA of nanowire targets by filling the gaps with prescribed low-density plasma. They found that the prescribed plasma can inhibit laser propagation in the nanowires and significantly reduce the proton energy. In the discussions above, the effect of the pre-pulse is not taken into consideration. Here, a set of simulations were carried out to briefly investigate the influence of pre-plasma. The density and profile of the pre-plasma are influenced by many factors such as the contrast of the laser pulse and target material. However, in typical experiments on nanowire targets, the requirement for the contrast of the laser pulse is rather strict, even above $1 \times 10^{12}$ [23,39]. For simplicity, we set the density of pre-plasma as $n_{c}$ and distributed it uniformly in the gaps. The simulation parameters are identical to those introduced in Section 2, except that the gaps are filled with plasma with an electron density of $1 n_{c}$ to represent the pre-plasma created by a laser pre-pulse. The energy spectra of the protons at the end of the simulation are shown in Figure 6a. The CNT can still generate much more energetic protons than the PNT. Furthermore, compared to the cases without pre-plasma, the cut-off energies of the protons are only slightly decreased, meaning that the influence of pre-plasma is not significant. This can be understood from the laser propagation in under-critical plasma. The non-relativistic critical density is $n_{\mathcal{c}}$, while for a laser pulse with large intensity, this value should thus be modified as $\gamma n_{\mathcal{c}}$, where $\gamma=\sqrt{1+a_{0}^{2}}$ is the relativistic factor [40]. In our case, $a_{0}=8$, and the corresponding relativistic critical density is approximately $8 n_{\mathcal{c}}$, much larger than the density of filled pre-plasma. This means that the laser pulse can propagate through the gaps, and the pre-plasma does not have much influence on the absorption. Figure $6 \mathrm{~b}, \mathrm{c}$ present the electron density at $t=22 T_{0}$, where the main pulse has completely interacted with the target and generated a large volume of hot electrons near the target front. One can see that the generated hot electrons fill in the gaps, 
and their density is as much as $10 n_{c}$, beyond the relativistic critical density. These electrons play the main role in reflecting the laser pulse, and the pre-plasma is thus negligible.
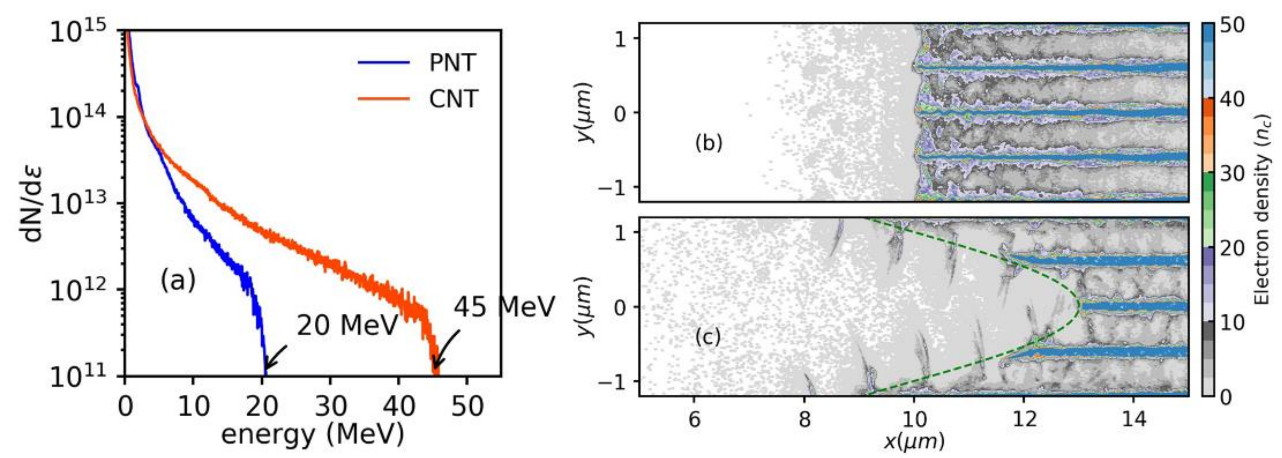

Figure 6. (a) Energy spectra of protons at the end of simulation. (b,c) Density of electrons at $t=22 T_{0}$.

The fill factor $\left(f=\frac{d_{1}}{d_{1}+d_{2}}\right)$ is also an important factor in the nanowire target, which significantly influences the absorption of the laser pulse and cutoff energies of accelerated protons $[29,41]$. The performance of the CNT and the PNT at different fill factors were also briefly investigated. Figure 7 shows the cutoff energies of the protons. The CNT can accelerate protons to higher energies than the PNT with different fill factors. However, when the fill factor is relatively small, i.e., the density of the nanowires is low, the enhancement of the CNT is not as significant as in the cases with higher fill factors. This is because in the cases with small fill factors, the reflectivity of the laser pulse at the tips of the PNT is naturally lower, and the corrugated surface can only slightly suppress the reflectivity. Furthermore, the multi-reflection and focusing of the laser pulse are also reduced due to the smaller area of interaction near the target front.

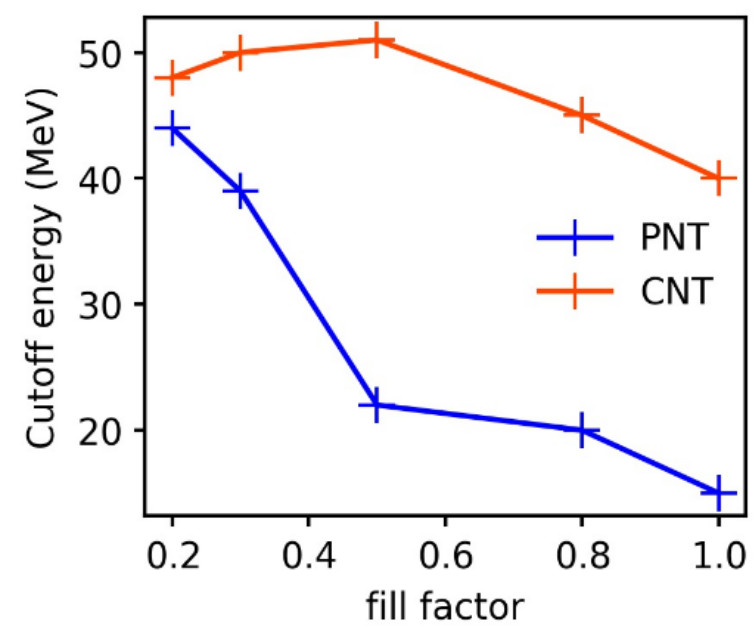

Figure 7. Cutoff energies of protons versus fill factors for the PNT and the CNT.

\section{Conclusions}

In summary, a novel nanowire target with a corrugated front surface was proposed to improve the proton acceleration via TNSA. Two-dimensional particle-in-cell simulations demonstrated that this target design can significantly increase the cut-off energy, the laser-toproton energy conversion efficiency and the number of accelerated protons, compared to an ordinary nanowire target. This enhancement can be attributed to suppressed reflectivity and enhanced absorption by the corrugated front surface. Furthermore, a series of simulations were carried out with various laser and target parameters to verify the robustness of the CNT. The simulation results show that the CNT always has better performance than the PNT and is robust with different simulation parameters. Given the technological advances 
in the fabrication of nano-structured targets, our scheme may provide insight for obtaining high-energy proton beams without requiring higher laser intensity.

Author Contributions: Conceptualization, simulation, data analysis and writing-original draft preparation, Y.C.; writing - review and editing, L.C., C.Z. and Z.L.; project administration, X.H. All authors have read and agreed to the published version of the manuscript.

Funding: This research was funded by the National Natural Science Foundation of China, grant number 11875091 and 11975059.

Institutional Review Board Statement: Not applicable.

Informed Consent Statement: Not applicable.

Data Availability Statement: The data that support the findings of this study are available from the corresponding author upon reasonable request.

Acknowledgments: The EPOCH code is used under UK EPSRC contract (EP/G055165/1 and EP/G056803/1). Yue Chao would like to thank Rui Xie, Yan Jiang and Xinxin Yan for useful discussions and help.

Conflicts of Interest: The authors declare no conflict of interest.

\section{References}

1. Daido, H.; Nishiuchi, M.; Pirozhkov, A.S. Review of Laser-Driven Ion Sources and Their Applications. Rep. Prog. Phys. 2012, 75, 056401. [CrossRef]

2. Borghesi, M.; Mackinnon, A.J.; Campbell, D.H.; Hicks, D.G.; Kar, S.; Patel, P.K.; Price, D.; Romagnani, L.; Schiavi, A.; Willi, O. Multi-MeV Proton Source Investigations in Ultraintense Laser-Foil Interactions. Phys. Rev. Lett. 2004, 92, 055003. [CrossRef]

3. Schardt, D. Tumor Therapy with High-Energy Carbon Ion Beams. Nucl. Phys. A 2007, 787, 633-641. [CrossRef]

4. Patel, P.; Mackinnon, A.; Key, M.; Cowan, T.; Foord, M.; Allen, M.; Price, D.; Ruhl, H.; Springer, P.; Stephens, R. Isochoric Heating of Solid-Density Matter with an Ultrafast Proton Beam. Phys. Rev. Lett. 2003, 91, 125004. [CrossRef]

5. Palaniyappan, S.; Huang, C.; Gautier, D.C.; Hamilton, C.E.; Santiago, M.A.; Kreuzer, C.; Sefkow, A.B.; Shah, R.C.; Fernández, J.C. Efficient Quasi-Monoenergetic Ion Beams from Laser-Driven Relativistic Plasmas. Nat. Commun. 2015, 6, 10170. [CrossRef] [PubMed]

6. Zhang, F.; Cai, H.B.; Zhou, W.M.; Dai, Z.S.; Shan, L.Q.; Xu, H.; Chen, J.B.; Ge, F.J.; Tang, Q.; Zhang, W.S.; et al. Enhanced Energy Coupling for Indirect-Drive Fast-Ignition Fusion Targets. Nat. Phys. 2020, 16, 810-814. [CrossRef]

7. Tabak, M.; Hammer, J.; Glinsky, M.E.; Kruer, W.L.; Wilks, S.C.; Woodworth, J.; Campbell, E.M.; Perry, M.D.; Mason, R.J. Ignition and High Gain with Ultrapowerful Lasers*. Phys. Plasmas 1994, 1, 1626-1634. [CrossRef]

8. Snavely, R.A.; Key, M.H.; Hatchett, S.P.; Cowan, T.E.; Roth, M.; Phillips, T.W.; Stoyer, M.A.; Henry, E.A.; Sangster, T.C.; Singh, M.S.; et al. Intense High-Energy Proton Beams from Petawatt-Laser Irradiation of Solids. Phys. Rev. Lett. 2000, 85, 2945-2948. [CrossRef]

9. Wilks, S.C.; Langdon, A.B.; Cowan, T.E.; Roth, M.; Singh, M.; Hatchett, S.; Key, M.H.; Pennington, D.; MacKinnon, A.; Snavely, R.A. Energetic Proton Generation in Ultra-Intense Laser-Solid Interactions. Phys. Plasmas 2001, 8, 542-549. [CrossRef]

10. Qiao, B.; Zepf, M.; Borghesi, M.; Geissler, M. Stable GeV Ion-Beam Acceleration from Thin Foils by Circularly Polarized Laser Pulses. Phys. Rev. Lett. 2009, 102, 145002. [CrossRef] [PubMed]

11. Henig, A.; Steinke, S.; Schnürer, M.; Sokollik, T.; Hörlein, R.; Kiefer, D.; Jung, D.; Schreiber, J.; Hegelich, B.M.; Yan, X.Q.; et al. Radiation-Pressure Acceleration of Ion Beams Driven by Circularly Polarized Laser Pulses. Phys. Rev. Lett. 2009, 103, 245003. [CrossRef] [PubMed]

12. Gonzalez-Izquierdo, B.; Capdessus, R.; King, M.; Gray, R.J.; Wilson, R.; Dance, R.J.; McCreadie, J.; Butler, N.M.H.; Hawkes, S.J.; Green, J.S.; et al. Radiation Pressure-Driven Plasma Surface Dynamics in Ultra-Intense Laser Pulse Interactions with Ultra-Thin Foils. Appl. Sci. 2018, 11, 336. [CrossRef]

13. Silva, L.O.; Marti, M.; Davies, J.R.; Fonseca, R.A.; Ren, C.; Tsung, F.S.; Mori, W.B. Proton Shock Acceleration in Laser-Plasma Interactions. Phys. Rev. Lett. 2004, 92, 015002. [CrossRef] [PubMed]

14. Fiuza, F.; Stockem, A.; Boella, E.; Fonseca, R.A.; Silva, L.O.; Haberberger, D.; Tochitsky, S.; Gong, C.; Mori, W.B.; Joshi, C. Laser-Driven Shock Acceleration of Monoenergetic Ion Beams. Phys. Rev. Lett. 2012, 109, 215001. [CrossRef] [PubMed]

15. Wagner, F.; Deppert, O.; Brabetz, C.; Fiala, P.; Kleinschmidt, A.; Poth, P.; Schanz, V.A.; Tebartz, A.; Zielbauer, B.; Roth, M.; et al. Maximum Proton Energy above $85 \mathrm{MeV}$ from the Relativistic Interaction of Laser Pulses with Micrometer Thick CH 2 Targets. Phys. Rev. Lett. 2016, 116, 205002. [CrossRef]

16. Wagner, F.; Bedacht, S.; Bagnoud, V.; Deppert, O.; Geschwind, S.; Jaeger, R.; Ortner, A.; Tebartz, A.; Zielbauer, B.; Hoffmann, D.H.H.; et al. Simultaneous Observation of Angularly Separated Laser-Driven Proton Beams Accelerated via Two Different Mechanisms. Phys. Plasmas 2015, 22, 063110. [CrossRef] 
17. Honrubia, J.J.; Morace, A.; Murakami, M. On Intense Proton Beam Generation and Transport in Hollow Cones. Matter Radiat. Extrem. 2017, 2, 28-36. [CrossRef]

18. Sgattoni, A.; Londrillo, P.; Macchi, A.; Passoni, M. Laser Ion Acceleration Using a Solid Target Coupled with a Low-Density Layer. Phys. Rev. E 2012, 85, 036405. [CrossRef]

19. Yang, Y.C.; Zhou, C.T.; Huang, T.W.; Ju, L.B.; Jiang, K.; Cai, T.X.; Zhang, H.; Wu, S.Z.; Qiao, B.; Yu, M.Y.; et al. Proton Acceleration from Laser Interaction with a Complex Double-Layer Plasma Target. Phys. Plasmas 2018, 25, 123107. [CrossRef]

20. Prencipe, I.; Sgattoni, A.; Dellasega, D.; Fedeli, L.; Cialfi, L.; Choi, I.W.; Kim, I.J.; Janulewicz, K.A.; Kakolee, K.F.; Lee, H.W.; et al. Development of Foam-Based Layered Targets for Laser-Driven Ion Beam Production. Plasma Phys. Control. Fusion 2016, 58, 034019. [CrossRef]

21. Blanco, M.; Flores-Arias, M.T.; Ruiz, C.; Vranic, M. Table-Top Laser-Based Proton Acceleration in Nanostructured Targets. New J. Phys. 2017, 19, 033004. [CrossRef]

22. Chatterjee, G.; Singh, P.K.; Ahmed, S.; Robinson, A.P.L.; Lad, A.D.; Mondal, S.; Narayanan, V.; Srivastava, I.; Koratkar, N.; Pasley, J.; et al. Macroscopic Transport of Mega-Ampere Electron Currents in Aligned Carbon-Nanotube Arrays. Phys. Rev. Lett. 2012, 108, 235005. [CrossRef] [PubMed]

23. Moreau, A.; Hollinger, R.; Calvi, C.; Wang, S.; Wang, Y.; Capeluto, M.G.; Rockwood, A.; Curtis, A.; Kasdorf, S.; Shlyaptsev, V.N.; et al Enhanced Electron Acceleration in Aligned Nanowire Arrays Irradiated at Highly Relativistic Intensities. Plasma Phys. Control. Fusion 2020, 62, 014013. [CrossRef]

24. Xiao, K.D.; Huang, T.W.; Zhou, C.T.; Qiao, B.; Wu, S.Z.; Ruan, S.C.; He, X.T. Enhanced Target Normal Sheath Acceleration of Protons from Intense Laser Interaction with a Cone-Tube Target. AIP Adv. 2016, 6, 015303. [CrossRef]

25. Brunel, F. Not-so-Resonant, Resonant Absorption. Phys. Rev. Lett. 1987, 59, 4. [CrossRef] [PubMed]

26. Calestani, D.; Villani, M.; Cristoforetti, G.; Brandi, F.; Koester, P.; Labate, L.; Gizzi, L.A. Fabrication of ZnO-Nanowire-Coated Thin-Foil Targets for Ultra-High Intensity Laser Interaction Experiments. Matter Radiat. Extrem. 2021, 6, 046903. [CrossRef]

27. Mondal, S. Aligned Copper Nanorod Arrays for Highly Efficient Generation of Intense Ultra-Broadband THz Pulses. Sci. Rep. 2017, 7, 1-8. [CrossRef]

28. Jiang, S.; Ji, L.L.; Audesirk, H.; George, K.M.; Snyder, J.; Krygier, A.; Poole, P.; Willis, C.; Daskalova, R.; Chowdhury, E.; et al Microengineering Laser Plasma Interactions at Relativistic Intensities. Phys. Rev. Lett. 2016, 116, 085002. [CrossRef] [PubMed]

29. Dozières, M.; Petrov, G.M.; Forestier-Colleoni, P.; Campbell, P.; Krushelnick, K.; Maksimchuk, A.; McGuffey, C.; Kaymak, V.; Pukhov, A.; Capeluto, M.G.; et al. Optimization of Laser-Nanowire Target Interaction to Increase the Proton Acceleration Efficiency. Plasma Phys. Control. Fusion 2019, 61, 065016. [CrossRef]

30. Margarone, D.; Klimo, O.; Kim, I.J.; Prokůpek, J.; Limpouch, J.; Jeong, T.M.; Mocek, T.; Pšikal, J.; Kim, H.T.; Proška, J.; et al. Laser-Driven Proton Acceleration Enhancement by Nanostructured Foils. Phys. Rev. Lett. 2012, 109, 234801. [CrossRef]

31. Arber, T.D.; Bennett, K.; Brady, C.S.; Lawrence-Douglas, A.; Ramsay, M.G.; Sircombe, N.J.; Gillies, P.; Evans, R.G.; Schmitz, H.; Bell, A.R.; et al. Contemporary Particle-in-Cell Approach to Laser-Plasma Modelling. Plasma Phys. Control. Fusion 2015, 57, 113001. [CrossRef]

32. Hollinger, R.; Wang, S.; Wang, Y.; Moreau, A.; Capeluto, M.G.; Song, H.; Rockwood, A.; Bayarsaikhan, E.; Kaymak, V.; Pukhov, A.; et al. Extreme Ionization of Heavy Atoms in Solid-Density Plasmas by Relativistic Second-Harmonic Laser Pulses. Nat. Photonics 2020, 14, 607-611. [CrossRef]

33. He, H.; Qiao, B.; Shen, X.F.; Yao, W.P.; Xie, Y.; Zhou, C.T.; He, X.T.; Zhu, S.P.; Pei, W.B.; Fu, S.Z. High-Flux High-Energy Ion Beam Production from Stable Collisionless Shock Acceleration by Intense Petawatt-Picosecond Laser Pulses. New J. Phys. 2019, 21, 033035. [CrossRef]

34. He, H.; Qiao, B.; Shen, X.F.; Yao, W.P.; Yao, Y.L.; Zhou, C.T.; He, X.T.; Zhu, S.P.; Pei, W.B.; Fu, S.Z. All-Optical Cascaded Ion Acceleration in Segmented Tubes Driven by Multiple Independent Laser Pulses. Plasma Phys. Control. Fusion 2019, $61,115005$. [CrossRef]

35. Cao, L.; Gu, Y.; Zhao, Z.; Cao, L.; Huang, W.; Zhou, W.; Cai, H.B.; He, X.T.; Yu, W.; Yu, M.Y. Control of the Hot Electrons Produced by Laser Interaction with Nanolayered Target. Phys. Plasmas 2010, 17, 103106. [CrossRef]

36. Gizzi, L.A.; Cristoforetti, G.; Baffigi, F.; Brandi, F.; D'Arrigo, G.; Fazzi, A.; Fulgentini, L.; Giove, D.; Koester, P.; Labate, L.; et al. Intense Proton Acceleration in Ultrarelativistic Interaction with Nanochannels. Phys. Rev. Res. 2020, 2, 033451. [CrossRef]

37. Zou, D.B.; Pukhov, A.; Yi, L.Q.; Zhuo, H.B.; Yu, T.P.; Yin, Y.; Shao, F.Q. Laser-Driven Ion Acceleration from Plasma Micro-Channel Targets. Sci. Rep. 2017, 7, 42666. [CrossRef]

38. Mora, P. Plasma Expansion into a Vacuum. Phys. Rev. Lett. 2003, 90, 185002. [CrossRef]

39. Eftekhari-Zadeh, E.; Blümcke, M.S.; Samsonova, Z.; Loetzsch, R.; Uschmann, I.; Zapf, M.; Ronning, C.; Rosmej, O.N.; Kartashov, D.; Spielmann, C. Laser Energy Absorption and X-Ray Generation in Nanowire Arrays Irradiated by Relativistically Intense UltraHigh Contrast Femtosecond Laser Pulses. Phys. Plasmas 2022, 29, 013301. [CrossRef]

40. Wilks, S.C.; Kruer, W.L.; Tabak, M.; Langdon, A.B. Absorption of Ultra-Intense Laser Pulses. Phys. Rev. Lett. 1992, 69, 1383-1386. [CrossRef]

41. Xie, R.; Cao, L.H.; Chao, Y.; Jiang, Y.; Liu, Z.J.; Zheng, C.Y.; He, X.T. Improvement of Laser Absorption and Control Ofparticle Acceleration by Subwavelength Nanowire Target. Phys. Plasmas 2020, 27, 123108. [CrossRef] 